\title{
Ab Initio Lattice Dynamics of $\mathbf{M g B}_{2}$
}

\author{
K. Parlinski \\ Institute of Nuclear Physics, Radzikowskiego 152, 31-342 Cracow, Poland \\ Using the density functional theory, the phonon dispersion relations, \\ the phonon density of states, mean square displacements, and thermody- \\ namic functions of superconducting $\mathrm{MgB}_{2}$ crystals have been calculated. \\ The modes of graphite-like boron network belong to optic phonon branches, \\ which are rather independent of the $\mathrm{Mg}$ vibrations. \\ PACS numbers: 63.20.-e, 74.20.-Z
}

Recently, the discovery of superconductivity $\left(T_{\mathrm{c}} \sim 39 \mathrm{~K}\right)$ in hexagonal magnesium diboride $\mathrm{MgB}_{2}$ has been announced [1]. It is believed [2] that this material becomes superconducting by the classical BCS mechanism proposed by Bardeen, Cooper, and Schieffer. This mechanism assumes that the interaction between the electrons which give rise to superconductivity is mediated by thermal phonons in the underlying lattice.

First principles FLMTO-GGA electronic structure calculations $[3,4]$ showed that in superconducting $\mathrm{MgB}_{2}$ the high energy valence band is made predominantly of boron $2 p$-states which determine the electronic density of states in the vicinity of the Fermi surface. It has been already shown that the electronic density of states at the Fermi level $[3,4]$ and the conductivity in the boron plane [3] are quite substantial.

Modern $a b$ intio determination of phonon dispersion relations is the way to help the understanding of the role played by the electron-phonon coupling. Recently a number of contributions related to the lattice dynamics of $\mathrm{MgB}_{2}$ have been published, $[3,5-8]$. Some investigations were restricted to phonon modes for the $\Gamma$ point only $[3,5]$. In others, the phonon dispersions for $\mathrm{MgB}_{2}$ have been determined $[6,7]$. Present calculations offer additional information following from the knowledge of the lattice dynamics of $\mathrm{MgB}_{2}$.

The $a b$ initio calculations are carried out using the density functional theory with the generalized gradient approximation (GGA) as implemented in VASP package $[9,10]$, and with Vaderbilt-type ultrasoft pseudopotentials provided with this program. VASP solves the generalized Kohn-Sham equations by an iterative matrix diagonalization based on the minimization of the norm of the residuum vector to each eigenstate and an optimized charge-density mixing routine. The pseudopotentials for $\mathrm{B}$ and $\mathrm{Mg}$ atoms are representing $s^{2} p^{1}$ and $s^{2} p^{0}$ electron 
configurations, respectively. The summation over $k$ wave vectors was limitted to $12 \times 12 \times 12$ Monkhurst-Pack mesh. To optimize the crystal structure we used a primitive hexagonal unit cell, space group $P 6 / \mathrm{mmm}$ with 3 atoms at positions $\mathrm{Mg}:(0,0,0), \mathrm{B}:\left(\frac{1}{3}, \frac{2}{3}, \frac{1}{2}\right)$, and $\mathrm{B}:\left(\frac{2}{3}, \frac{1}{3}, \frac{1}{2}\right)$. This structure consists of layers of two-dimensional triangular lattice of either $\mathrm{Mg}$, intercalated by a two-dimensional planar graphite-like network of boron. The calculated structural parameters are $a=3.0651 \AA$ and $c=3.5176 \AA$, while the experimental values are $a=3.086 \AA$ and $c=3.524 \AA[1]$.

The phonons are determined by the direct method [11-14]. For that we optimized the rhombohedral supercell with 36 atoms and with basis vectors defined as $\boldsymbol{a}_{\mathrm{s}}=2 \boldsymbol{a}+\boldsymbol{c}, \boldsymbol{b}_{\mathrm{s}}=2 \boldsymbol{b}+\boldsymbol{c}, \boldsymbol{c}_{\mathrm{s}}=-2 \boldsymbol{a}-2 \boldsymbol{b}+\boldsymbol{c}$, where $\boldsymbol{a}, \boldsymbol{b}, \boldsymbol{c}$ are the primitive translation vectors of the hexagonal unit cell. The symmetry of the selected rhombohedral supercell breaks the twofold symmetry axis, and lowers the $P 6 / m m m\left(D_{6 h}^{1}\right)$ space group to $R \overline{3} m\left(D_{3 d}^{5}\right)$. The Hellmann-Feynman forces are computed for independent displacements $x, y$, and $z$ of metal and $\mathrm{B}$ atoms. The displacement amplitudes were varing between $0.03-0.30 \AA$, so that all the on-site Hellmann-Feyman forces were of the same order of magnitudes. To rebuild the correct symmetry of the phonon dispersion relations we use the same method as for the hexagonal $2 \mathrm{H}-\mathrm{GaN}$ crystal [13]. The corrections are so small that they are not visible on the figures. In this procedure we take into account 17 coordination shells of the force constants which required 64 independent parameters. The values of the force constants diminish with a distance between the involved atoms. The largest ones are the on-site force constants (at a distance zero). However, at the distance supercell center - supercell surface the largest elements are at least two order of magnitudes smaller than the on-site force constants. Such a decrease in the force constants is quite sufficient to obtain phonon frequencies at all wave vectors with appreciate accuracy. Moreover, the present rhombohedral supercell provides correct phonon frequencies at commensurate wave vectors $\Gamma:(0,0,0), M$ : $\left(0, \frac{1}{2}, 0\right),\left(\frac{1}{6}, \frac{1}{6}, \frac{1}{3}\right)$, and $\left(\frac{1}{3}, \frac{1}{3}, \frac{1}{3}\right)$ and symmetry equivalent points, even if the force constants would not vanish within the range of the supercell.

Let us return again to the distance dependence of the force constants. In Table I we show the three eigenvalues of the symmetrized force constant matrices for the coordination shells of the shortest radius $R$ around the metal and boron atoms. These force constants provide the largest contributions to the dynamical matrices. From Table I one can see that the force constants between nearest neighbors of boron $\mathrm{B}-\mathrm{B}$ are the largest. The force constants between boron and magnesium $\mathrm{B}-\mathrm{Mg}$ and between magnesiums $\mathrm{Mg}-\mathrm{Mg}$ prove to be much smaller. These features cause that the graphite-like network of borons vibrates rather independently on the magnesium sublattice.

In Fig. 1, we show the phonon dispersion relations for $\mathrm{MgB}_{2}$. In Table II the $\Gamma$-point phonon frequencies are collected. They are in reasonable agreement with other calculations $[3,5-7]$. Far from the $\Gamma$-point the acoustic phonon dispersion 
TABLE I

The quantities $\eta_{i} / \Phi_{i i}$, where $\eta_{i}, i=1,2,3$ are the eigenvalues of the symmetrized part of the force constant matrices for the coordination shells of the radius $R$ expressed in terms of respective diagonal on-site force constants $\Phi_{i i}$ of B atoms. The values of the on-site force constants of boron are as follows: $\Phi_{11}(0 ; 0)=\Phi_{22}(0 ; 0)=264 \mathrm{~N} / \mathrm{m}$, and $\Phi_{33}(0 ; 0)=156 \mathrm{~N} / \mathrm{m}$. The $\mathrm{Mg}(0)$ denotes the on-site force constant of the metal. Only quantities $\eta_{i} / \Phi_{i i}$ of the shortest radius $R$ are given.

\begin{tabular}{c|c|c|c|c|c}
\hline \hline Crystal & Bond & $R$ in $\AA$ & $\eta_{1}$ & \multicolumn{1}{c|}{$\eta_{2}$} & \multicolumn{1}{c}{$\eta_{3}$} \\
\hline $\mathrm{MgB}_{2}$ & $\mathrm{Mg}(0)$ & 0.000 & 0.390 & 0.390 & 0.612 \\
& $\mathrm{~B}-\mathrm{B}$ & 1.770 & -0.123 & -0.232 & -0.246 \\
& $\mathrm{~B}-\mathrm{Mg}$ & 2.495 & -0.062 & 0.004 & -0.009 \\
& $\mathrm{~B}-\mathrm{B}$ & 3.065 & -0.138 & -0.014 & 0.038 \\
& $\mathrm{Mg}-\mathrm{Mg}$ & 3.065 & -0.044 & 0.003 & 0.009
\end{tabular}

\section{TABLE II}

Comparison of the $\Gamma$-point phonon frequencies (in $\mathrm{THz}$ ) of $\mathrm{MgB}_{2}$ with those of Refs. [3, 5-7] denoted as (I), (II), (III), (IV), respectively.

\begin{tabular}{c|c|c|c|c|c}
\hline \hline Mode & \multicolumn{1}{|c|}{ I } & \multicolumn{1}{|c|}{ II } & III & \multicolumn{1}{c|}{ IV } & Ours \\
\hline$E_{1 \mathrm{u}}$ & 9.8 & 9.6 & 10.0 & 9.7 & 9.4 \\
$A_{2 \mathrm{u}}$ & 11.6 & 11.7 & 12.0 & 11.8 & 10.9 \\
$E_{2 \mathrm{~g}}$ & 19.9 & 14.1 & 17.6 & 16.1 & 15.0 \\
$B_{1 \mathrm{~g}}$ & 20.4 & 20.7 & 20.8 & 20.9 & 20.8
\end{tabular}

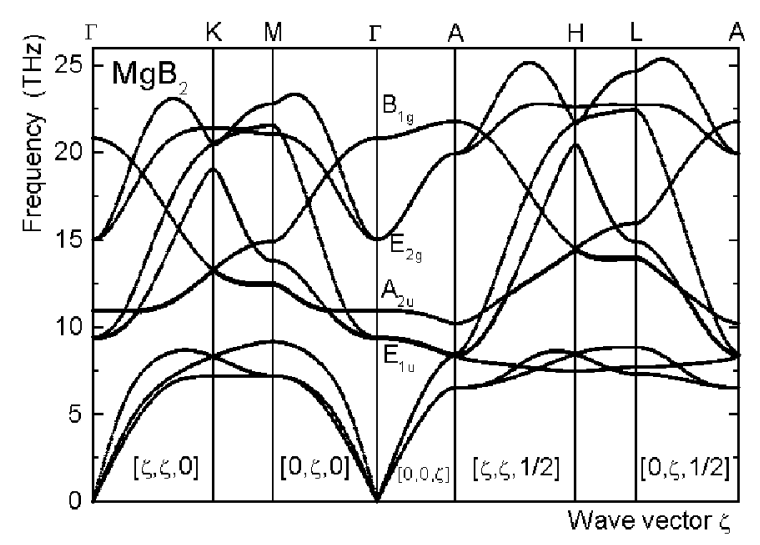

Fig. 1. Phonon dispersion relations of $\mathrm{MgB}_{2}$ crystal.

relations reflect the vibrations of the metals $\mathrm{Mg}$. The optic branches describe the vibrations of the graphic-like boron network. Moreover, the branches of symmetry 
$A_{2 \mathrm{u}}$ and $B_{1 \mathrm{~g}}$ at the $\Gamma$-point correspond to out-of-plane boron vibrations, while four remaining curves with symmetry $E_{1 \mathrm{u}}$ and $E_{2 \mathrm{~g}}$ at the $\Gamma$-point determine the in-plane boron motion. The out-of-plane and in-plane polarizations are approximately kept along the same branches all over the Brillouin zone. This feature could justify a construction of a simple two-dimensional model for either out-of-plane, or in-plane phonon boron vibrations. Notice that the in-plane boron modes $E_{2 \mathrm{~g}}$ and $E_{1 \mathrm{u}}$ exhibit a considerable dispersion over the Brillouin zone. The $E_{2 \mathrm{~g}}$ mode shows an exceptionally strong coupling to electronic states at the Fermi level [7]. The decouplings of the magnesium and boron vibrations to acoustic and optic modes, respectively, are caused by the large mass difference of the constituent atoms.

Figure 2 shows the total and partial phonon density of states. The partial phonon density of states is separated to modes with vibrational polarization within the graphite-like network plane, and out of it.

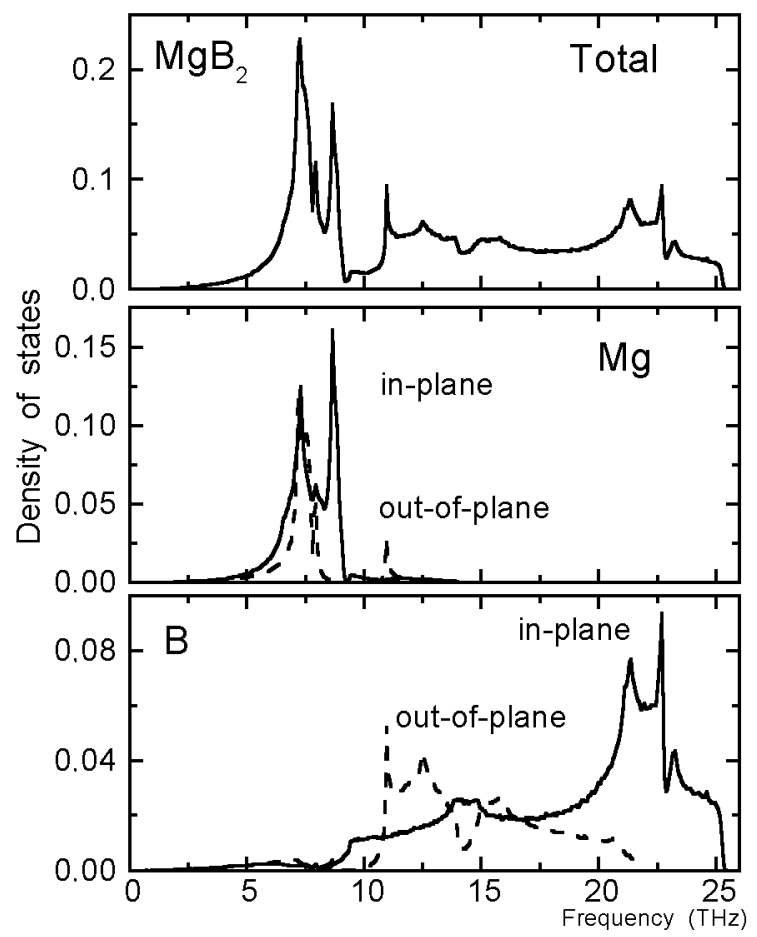

Fig. 2. Total and partial phonon density of states of $\mathrm{MgB}_{2}$ crystals. The magnesium and boron partial phonon density of states correspond to in-plane (solid line) and out-of-plane (dotted line) vibrations.

$g(\omega)$ allows us to determine the mean square displacements of atoms $B_{i j}=\left\langle u_{i} u_{j}\right\rangle ; B_{i j}$ defines, in turn, the Debye-Waller factor refined during the structural analysis. We have calculated these values and for two temperatures 
TABLE III

The calculated mean square displacements $B_{i j}=\left\langle u_{i} u_{j}\right\rangle$ are in units of $10^{-2} \AA^{2}$. Experimental data are taken from Ref. [15].

\begin{tabular}{c|c|c|c|c|c}
\hline \hline \multirow{2}{*}{ Temp. } & \multirow{2}{*}{$B_{i, j}$} & \multicolumn{2}{|c|}{ Calculations } & \multicolumn{2}{c}{ Experiment } \\
\cline { 3 - 6 } & & $\mathrm{Mg}$ & $\mathrm{B}$ & $\mathrm{Mg}$ & $\mathrm{B}$ \\
\hline $0^{\circ} \mathrm{K}$ & $B_{x x}$ & 0.28 & 0.28 & 0.31 & 0.34 \\
& $B_{z z}$ & 0.29 & 0.36 & 0.33 & 0.44 \\
\hline $300^{\circ} \mathrm{K}$ & $B_{x x}$ & 0.56 & 0.37 & 0.55 & 0.45 \\
& $B_{z z}$ & 0.61 & 0.53 & 0.56 & 0.65
\end{tabular}

they are listed in Table III. Also a comparison of the calculated $B_{i j}$ and the measured [15] data is given there. For Mg the agreement is perfect, while for B the measured values are slightly higher, which might mean that a small static displacement of the B atoms persists in the crystal.

Figure 3 shows the temperature dependence of internal energy, free energy, entropy, and heat capacity at a constant volume in harmonic approximation. The limit of the internal energy, and the free energy at $T=0$ are equal to the mean

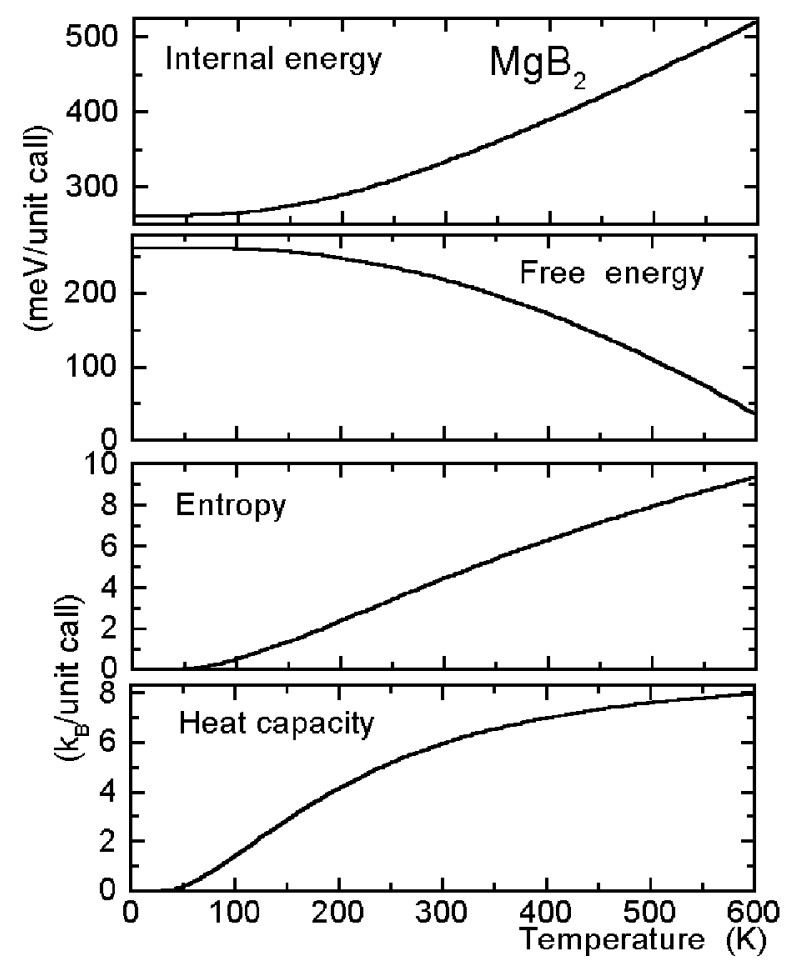

Fig. 3. Internal energy, free energy, entropy, and heat capacity at a constant volume as a function of temperature for $\mathrm{MgB}_{2}$ in harmonic approximation. 
energy of zero mode frequencies. At high temperatures the internal energy tends to $k_{\mathrm{B}} T$ behavior. The heat capacity tends at high temperatures to $9 k_{\mathrm{B}}$, where 9 is the number of degree of freedom in the unit cell of $\mathrm{MgB}_{2}$. This regime is attained above $1600 \mathrm{~K}$.

We have performed structural calculations for $\mathrm{MgB}_{2}$ crystal, and derived the phonon dispersion relations, phonon density of states, mean-square displacement factors, and thermodynamic functions. We found that $\mathrm{B}$ atoms appear to be strongly bounded within the graphite-like network suggesting a substantial two-dimensionality of these compounds. Generally, the magnesium vibrations are limited to a low frequency interval.

The author would like to thank S. Kaprzyk and A.M. Oleś for fruitful discussions. This work was partially supported by the State Committee for Scientific Research, grant No. 5 PO3B 06920.

\section{References}

[1] J. Nagamatsu, N. Nakagawa, T. Muranaka, Y. Zenitani, J. Akimitsu, Nature 410, 63 (2001)

[2] J. Cava, Nature 410, 23 (2001).

[3] G. Satta, G. Profeta, F. Bernardini, A. Continenza, S. Massidoka, Phys. Rev. B, in print.

[4] N.I. Medvedeva, A.L. Ivanovskii, J.E. Medvedeva, A.J. Freeman, Phys. Rev. B 64, 20502 (2001).

[5] J. Kortus, I.I. Mazin, K.D. Belashchenko, V.P. Antropov, Phys. Rev. Lett. 86, 4656 (2001).

[6] Y. Kong, O.V. Dolgov, O. Jepsen, O.K. Andersen, Phys. Rev. B 64, 020501 (2001).

[7] K.-P. Bohnen, R. Heid, B. Renker, Phys. Rev. Lett. 86, 5771 (2001).

[8] R. Osborn, E.A. Goremychkin, A.I. Kolesnikov, D.G. Hinks, Phys. Rev. Lett. 87, 017005 (2001).

[9] G. Kresse, J. Hafner, Phys.Rev. B 47, 558 (1993); ibid 49, 14251 (1994).

[10] G. Kresse, J. Furhmüller, Software VASP, Vienna (1999); Phys.Rev. B 54, 11169 (1996); Comput. Mat. Science 6, 15 (1996).

[11] K. Parlinski, Z.Q. Li, Y. Kawazoe, Phys. Rev. Lett. 78, 4063 (1997).

[12] K. Parlinski, in: Neutrons and Numerical Methods $N_{2} M$, Eds. M.R. Johnson, G.J. Kearley, H.G. Büttner, in series Am. Inst. Phys. Conf. Proc., Vol. 479, AIP, Woodbury (N.Y.) 1999, p. 121

[13] K. Parlinski, Y. Kawazoe, Phys. Rev. B 60, 15511 (1999).

[14] K. Parlinski, Software PHONON, http://wolf.ifj.edu.pl./phonon/(2001).

[15] J.D. Jorgensen, D.G. Hinks, S. Short, Phys. Rev. B 63, 224522 (2001). 